\title{
A SYSTEMATIC APPROACH TO THE QUALITATIVE META-SYNTHESIS
}

\author{
Philip D. Noah Jr.,philipdnoah@gmail.com
}

\begin{abstract}
In the past fifty years, the number of published research papers has grown at an almost exponential rate, while at the same time the use of qualitative methodologies has gained popularity. Quantitative researchers were quick to grasp the value of being able to combine, or synthesis the results from serval small studies into a larger, more comprehensive study called a meta-analysis. In the late 1990's researchers in the fields of education and health care applied the concept of the meta-analysis to qualitative research. The qualitative meta-synthesis uses secondary data in an attempt to create a "holistic interpretation" and uncover new knowledge as opposed to simply aggregating numbers or presenting a review of the literature (Jensen and Allan 1996; Noah, 2013). By taking a systematic approach to the qualitative meta-synthesis researchers have a method of combining qualitative data without losing the richness of the original works while providing an "audit trial" as well as "reproducibility" of results.
\end{abstract}

Keywords: Meta-Analysis, Meta-synthesis, Secondary Analysis, Qualitative Data, Research Methods, Interpretive, Meta-Study, Qualitative Meta-Synthesis

\section{INTRODUCTION}

"Your ability to tell a story based on the facts is as essential as your ability to assemble the facts. The point of research and analysis is not simply to collect data, but to provide a basis for solutions."

- Elizabeth Dole, 2014 Pardee RAND commencement address

\section{The need for meta-synthesis}

There has been an almost exponential growth in the number of published research studies over the last 50 years with few studies providing adequate answers to their primary research question (Weed, 2005). In response to the lack of answers, researchers conduct additional primary research in an attempt to overcome the shortcomings of pervious research and to fill in the gaps in the body of knowledge. Often times these attempts end with the conclusion that even more research is needed. This is why there is a need to combine the results of many studies into a new original piece of research. The question is how to combine the results from several qualitative studies in a manner that is systematic and reproducible? This paper examines one such approach, the Systematic Qualitative Meta-Synthesis.

A meta-synthesis uses existing research as the "data," extracts the "trends" and "principles" from them, and combines them to create new knowledge and new understandings of the topic. The strength of the meta-synthesis is the ability to find patterns of results that may not come to light through one study or even the review of a dozen studies (Glass, 1976). The meta-synthesis started out as a method for combining quantitative studies, but it was soon adapted to qualitative research by practitioners in education and nursing. Smith, Pope, and Botha (2005) studied why patients delay seeking medical attention for cancer through the use of a qualitative synthesis. The researchers used 32 qualitative peer reviewed papers that detailed the experiences of 775 people with 20 different types of cancer (Smith et. al., 2005). The use of a qualitative synthesis allowed for comparisons that were not made in other studies due to their small sample size and focus on gender-specific cancers (Smith et. al., 2005).

By developing a systematic approach to the qualitative meta-synthesis students and researchers in any field will have a method of conducting research that will produce reproducible, cumulative, and actionable results. The use of statistical analysis to explore the key concepts of the domain under study gives the qualitative meta-synthesis a mix- 
methods approach. The qualitative meta-synthesis enhances understanding while still keeping the human voice and story of the original works.

\section{LITERATURE REVIEW}

\section{Meta-Analysis}

Glass coined the term "meta-analysis" in 1976 in a paper titled Primary, Secondary, and meta-analysis of research. Glass used the tem meta-analysis "to refer to the statistical analysis of a large collection of analysis results from individual studies for the purpose of integrating the findings" (Glass, 1976, p3). Glass pointed to the rapidly growing volume of research in the education field as the catalyst for the meta-analysis. He likens the meta-analysis to examining 500 tests scores, looking at the raw scores does not tell you anything, it is only when they are analyzed and summarized via statistical analysis does the meaning become clear. Glass did not intend for the meta-analysis to be a simple summary or review of already conducted research. He saw it as a means to create new knowledge from existing information. The meta-analysis is a method to find patters that only become known when a large volume of studies is analyzed. For Glass the meta-analysis was a developing method of conducting original research using previously generated data. It is important to note that Glass argues for the inclusion of all relevant studies for a given problem without regard to the "quality" of the study. Glass bases this on his belief that there is very little difference between "poorly done" and "proper" studies and by rejecting poor studies valuable data can be lost. Glass states that even flawed studies may still have valid results and that the purpose of the meta-analysis is to "extract the message" from the research (Glass, 1976).

\section{Meta-synthesis}

The meta-synthesis is the qualitative equivalent of the meta-analysis, in that both methods use secondary data in an attempt to create a "holistic interpretation" and uncover new knowledge as opposed to simply aggregating numbers or presenting a review of the literature (Jensen and Allan 1996; Noah, 2013). The idea of the meta-synthesis was developed in the field of medicine and nursing by Jensen and Allan and in the education field by Turner. Jensen and Allan proposed a framework based on an "interpretive paradigm" with the goal of obtaining a "fuller knowledge" of the phenomenon under investigation (Jensen and Allan 1996). To create this fuller knowledge they suggested the use of comparative textual analysis, which results in a complete study (Jensen and Allan 1996). Jensen and Allan follow Glass in using the phenomenon under study as the criteria for the selection of data (Jensen and Allan 1996, Glass, 1976). Like Glass, Jensen and Allan also do not take the scientific merit of the research into account in order to avoid the exclusion of reverent studies (Jensen and Allan 1996). The interpretative process begins after the data collection phase. A first reading of the texts is conducted with the findings from each text being coded along with coding of the details relevant to the phenomenon under study (Jensen and Allan 1996). A list of key ideas, metaphors, phrases, and concepts is created (Jensen and Allan 1996). The final phase is the synthesis where the metaphors are translated and refined resulting in a description of the phenomenon under study (Jensen and Allan 1996). Jensen and Allan point out that the meta-synthesis must be grounded in the original data and must present a faithful interpretation of the experience such that "the people having the experience would immediately recognize it" (Jensen and Allan 1996, p. $556)$.

Turner who was writing at the same time as Jensen and Allan developed a definition of a qualitative secondary data analysis as being research done by someone who is "one removed" from the process using a different method than the original research to address the original question, or addressing a different research question (Turner 1997). From this definition, Turner identified three different approaches to conducting the qualitative research synthesis. The first method is the secondary analysis where existing qualitative data is re-examined using different methods to answer the original research question (Turner 1997). However, Weed points out that this approach can be problematic due to a lack of access to most research data performed by others (Weed 2005). The second method is meta-analysis. The metaanalysis of Turner is similar to the methods of Glass, and Jensen and Allan. Turner proposed the combining of the results of several studies, both published and unpublished, in order for them to be compared and synthesized to create new knowledge (Turner 1997). Turner calls the third method collaboration. In collaboration, the research involves external stakeholders as part of the process (Turner 1997). For example, a researcher using collaboration on the topic 
of nuclear waste would ask environmentalist, concerned citizens, policy makers, and industry representatives to contribute to the interruptive process (Turner 1997).

Meta-interpretation is a method of qualitative synthesis developed by Weed (2005). As the name suggest it is a method for conducting a qualitative synthesis based on interpretative epistemology. The meta-interpretation does not try to boil down qualitative research into quantitative data; rather it strives to maintain the "richness" of the original studies (Weed, 2005). The data for the meta-interpretation is the completed research (journal articles, dissertations, papers etc.) of other researchers. Weed (2005) points out this is done in order to keep the same interpretative epistemology as the original research and for a particle reason, lack of access to the primary data used for the original research. The meta-interpretation is based on five principles; an ideographic approach to exclusion criteria, meaning in context, interpretation as the raw data, an iterative approach to sampling, and a transparent audit trail (Weed, 2005). The metainterpretation, like a grounded theory study, starts with a research area instead of a research question (Weed, 2005). Once the research area is defined, the researcher selects studies for the first round of analysis. Weed (2005) suggests four or five studies selected using maximum variation sampling, however more may be needed to provide the researcher with a broad understanding of the research area (Noah, 2013). In the first round of analysis, the focus is on thematic and context analysis (Weed, 2005). From this analysis, the researcher finds common issues and themes that will be explored in further rounds of investigation. The studies used in the first round are then reviewed to see if any should be excluded. Weed (2005) suggests two main criteria for exclusion, flawed research or the study not being within bounds of the research topic (Weed, 2005). To maintain transparency and to provide an audit trail, the researcher should include in the finished research a detailed description of the exclusion criteria (Weed, 2005). The second phase of analysis is an iterative process that continues until data saturation occurs (Weed, 2005). More studies are identified and reviewed. The new studies are combined with the studies from the previous round and all studies then undergo thematic and context analysis. The exclusion criteria are also updated and studies from previous round are examine to see if they meet the new criteria for exclusion. Data saturation occurs when no new insights emerge (Weed, 2005). Once saturation occurs, the finds are written up along with a detailed description of the boundaries of the study and exclusion criteria (Weed, 2005). Weed (2005) cautions that the result of the meta-interpretation should not be that more research is needed, instead it should be synergetic. "The value of a synthesis [is]...the extent to which it produces insights that are more than the sum of the parts" (Wood, 2005 p.18).

Finlayson and Dixon (2008) build on the ideas of Jensen and Allen, that qualitative meta-synthesis should place an emphasis on the interpretation of findings. Finlayson and Dixon suggest that interpretation is not enough, qualitative meta-synthesis should "be added to the evidence base and used by policy makers and practitioners" (Finlayson and Dixon, 2008 p.60). The methodology proposed by Finlayson and Dixon for conducting a qualitative meta-synthesis is very similar to that of Jensen and Allan as well as Weed, but it differs in in its starting point. Whereas Jensen and Allan start with a phenomenon as the basis for the selection of data, Finlayson and Dixon take a more traditional approach by using a research question as the basis for focusing the data search (Finlayson and Dixon, 2008). The use of a research question makes Jensen and Allan's approach more appealing to the novice or student researcher who wishes to conduct a qualitative meta-synthesis, but needs to fit it within the framework of a traditional dissertation or thesis. Examples of the use of a research question to conduct a qualitative meta-synthesis based dissertation include Corbin-Staton (2009) in the field of education and Noah (2013) in information systems. Finlayson and Dixon, who were writing about research in the health field suggest that date ranges and inclusion and exclusion criteria be used to limit the scope of the synthesis and number of studies used. While giving no exact number of studies to include in the synthesis, Finlayson and Dixon note that synthesis have been performed with as few as four and as many as 292 (Finlayson and Dixon, 2008). On the issue of per-synthesis analysis of studies based on quality Finlayson and Dixon, agree with Jensen and Allan that excluding studies based on quality has little benefit and may lead to important findings being over looked. Finlayson and Dixon view the philosophical underpinnings of the qualitative metasynthesis as being holistic rather than reductionist. They emphasize the interpretive nature of the studies used in the synthesis as well as the need to create new insights and understandings. The result should be a guide for the public and policy makers and not just another interpretation of the literature. 


\section{CONDUCTING A QUALITATIVE META- SYNTHESIS; A SYSTEMATIC APPROACH}

\section{Proposed methodology}

We propose taking a systematic, top-down approach to the qualitative meta-synthesis in order to synthesis data in a way that is transparent and reproducible. The process starts with a broad search of the literature using key words and phrases. From this search, papers are selected for inclusion in the synthesis. The papers are examined and the large overarching themes are identified. Once the themes emerge, the papers are search again for key words that exemplify the theme. The number of occurrences of the key words is calculated and the top three for each theme is used as a basis for discovery of the "essence" of the issue or topic being researched. The results are presented in a narrative format to keep the "richness" and the "voice" of the original papers.

\section{Epistemology}

The qualitative meta-synthesis traces its root back to the quantitative meta-analysis as defined by Glass (1976). Glass who was working in the educational field defined the meta-analysis as "the analysis of analyses" (Glass, 1976 p.3). In the meta-analysis, several related research studies are combined to produce a larger collection of statistical results. The studies employ the same or very similar methods of analysis and similar variables that could be easily combined and analyzed for patterns. The patterns that result from the meta-analysis would be impossible to find by looking at just one study or even a dozen studies. The individual studies are the "information" and by combing, organizing, and summarizing the information new "knowledge" is created (Glass, 1976; Noah, 2013). This is very similar to the idea of BA where explicit (written) knowledge is combined with other explicit or tacit (non-written) knowledge to create a new set of knowledge. (Nonaka\& Konno, 1998, Borkovich \& Noah 2014). The meta-analysis was soon adopted by the medical field where researchers used it to combine smaller studies on drug and treatment options into more comprehensives reports with results from a larger population and/or broader demographics. At the same time, the medical field was adopting the meta-analysis a shift was taking place in the field of nursing where qualitative research was gain respect. The fields of nursing and psychology started to apply the ideas of Glass to qualitative research.

The qualitative meta-synthesis is similar to its quantitative counterpart the meta-analysis in that both methods produce new knowledge from the analysis and synthesis of previous research. They differ in that the qualitative meta-synthesis creates a "holistic interpretation" instead of an aggregation of quantities found in the quantitative meta-analysis (Finlayson \&Dixon, 2008; Jensen \& Allan, 1996; Turner, 1997; Weed, 2005). The purpose of the qualitative metasynthesis is to create new knowledge that is fuller and richer than that which can be found in the individual studies that make up the synthesis. A fuller knowledge is one based on evidence, not opinion, which is complete, and is actionable. The conclusion of a qualitative meta-synthesis should never be that more research is required.

\section{Define the study}

\section{Scope}

The scope of the study is defined by the phenomenon under study as proposed by Jensen and Allen as well as Weed or by the research question as proposed by Finlayson \& Dixon. In either case, the research question or phenomenon needs to be well defined and narrow enough so that the qualitative meta-synthesis can be conducted in a reasonable amount of time. If a broad topic is selected, such as in Newton (2011) where the phenomenon under study was the public's views on posthumous organ donation the scope can be limited in other ways such as published research between 1990 and 2008. The qualitative meta-synthesis should not be so narrow that it loses it inclusiveness; the voices of all stakeholders need to be heard. One should not forget that the purpose of the qualitative meta-synthesis is to produce actionable results.

\section{Appraisal criteria}

There is general agreement that a broad range of material should be reviewed when conducting a qualitative metasynthesis. The researcher should go beyond the standard of peer-reviewed journals to include dissertations, nonpublished works, trade publications, print, and web-media (Jensen and Allan 1996; Finlayson and Dixon, 2008; Suri 2011). The broad inclusion criterion helps to insure that a wide range of voices is heard. It also helps to address the 
problem of publication basis found in some academic fields. If the researcher decides to limit the scope of publications used, he should be transparent and explain how the research articles were selected and why there was a need to limit the scope.

\section{Conducting the primary search}

The primary search is the first pass at collecting data for the study. The purpose of the primary search is to uncover articles and other documents that are related to the topic under investigation. The first step in conducting the primary search is to develop a list of "key words" that relate to the research topic. In the primary search you want to "cast a wide net" in order to find as many documents as possible. To accomplish this you should use broad search terms as well as use multiple databases such as Ebsco and ProQuest, you should not limit your search to purely academic journals. In order to obtain the prospective of all possible stakeholders' documents such as newspapers, trade journals, dissertations, government papers, and industry white papers should be included.

In order to determine if the articles are within the scope of the research study the abstract or first few paragraphs of the document need to be read. It is up to the researcher to determine how relevant the document is to the study. Once the abstracts have been read a list of possible documents to include in the study is compiled. The number of documents needed to conduct the Qualitative Meta-Synthesis is subjective and depends on several factors including the topic under investigation, the time allocated to conduct the study, and when data saturation occurs. Data saturation is when additional documents do not reveal any new information. When data saturation is reached, it is advisable to look at other sources of data to reduce bias in the study. For example if data saturation occurs after searching academic journal databases, do a search of dissertations, government documents, and non-academic periodicals to see if new ideas and keywords appear. This helps to insure multiple stakeholders are heard from. There is no minimum or maximum number of documents needed, however you should keep in mind that not all documents found in the first round of searching will be useful. A good general guideline is between 25 to 100 documents.

\section{Primary analysis}

The primary analysis consists of carefully reading the documents selected in primary search in their entirety. The purpose of this phase is to determine if the document fits into the scope of the research. The question to ask is "is it relevant to the research topic?" While the academic ideal is to include only "high quality" journal articles Glass argues that even flawed studies may still have valid results (Glass 1976). The purpose of the meta-analysis is to "extract the message" from the research (Glass, 1976, Noah, 2013). It is recommended that the researcher provide an "audit trail" outlining the interpretations and decisions the researcher made during the synthesis as well as including the original interpretations of the studies used (Weed, 2005, Noah, 2013). During the primary analysis, the researcher may uncover topics and themes that were not explored when conducting the primary search. When this occurs, the researcher is encouraged to expand the original primary search and look for other documents on the new topic or theme. The search for documents should be a recursive process, ending only when no new themes emerge. During the primary analysis, the researcher should pay attention to the references and citations in the documents as a way to find more data. The papers referenced will often lead the researcher to the seminal works in the field. This is very similar to the idea of the snowball sampling method. It is only when all possible sources of data have been exhausted that the research moves on to the coding phase.

\section{Coding "Within case analysis"}

Coding for the qualitative meta-synthesis is similar to the analysis described by Creswell (2007) for the multiple case study approach. Creswell describes a two-stage analysis for multiple case studies where each case is examined for themes; this is called a within-case analysis (Creswell, 2007, Turner 1997). For the qualitative meta-synthesis we reread each article and look for major over-arching themes. The themes should exemplify the main ideas of the article, the problem or solution, the impact on stakeholders, or illustrates a main point. The researcher lists all themes for each article in the research study. The result is a list of themes or ideas. The number of themes or ideas will depend on the topic and the documents selected for inclusion. The ideal is to write down every theme until data saturation occurs. For example, Noah (2013) found that the overarching themes for the data analyzed relating to technology that is regulated and subsidized by the US government (Nuclear power, Cellular communications, and the proposed smart- 
gird) can be summarized as fitting into Economic factors, Public Policy, Technology, Government, and Safety/Security.

The next step in coding the documents is to go back to the documents and create an exhaustive list of words and short, two to three words, phrases that exemplify the theme. Once the list is created for the document a search is done to find how many times the word, referred to as a key word by Noah (2013), is found in the document. The number of occurrences is tallied in a table.

Table 1. In Case Analysis

Coding for The failed promise of nuclear power (Beaver, 2011).

\begin{tabular}{ll|ll|ll|}
\hline Economic & Freq. & Public policy & Freq. & Technology & Freq. \\
\hline Cost & 24 & Development & 16 & Technology & 24 \\
Construction & 22 & Public & 4 & Design & 18 \\
Commercial & 16 & Support & 4 & Experimental & 11 \\
Economy / Economic & 11 & Carbon & 2 & & \\
Competitive & 5 & Environmental & 2 & & \\
Finance & 3 & Private sector & 2 & & \\
Competition & 2 & Communities & 1 & & \\
Expensive & 2 & Moral obligation & 1 & \\
Funding & 2 & Public-private & 1 & \\
Opportunity & 2 & Strategy & 1 & & \\
Partnership & 2 & & & \\
Prices & 2 & & & \\
Profitability & 2 & & \\
\hline
\end{tabular}

This process is repeated resulting in a coding sheet for each document.

\section{Synthesis "Cross case analysis"}

The second stage of the analysis is called a cross-case analysis, in this stage the researcher looks for themes that are common across all the cases (Creswell, 2007, Yin 2009). Yin (2009) uses the term cross-case synthesis to describe a technique for examining two or more case studies by using word tables and looking for overall patterns. The word table as used by Yin is a simple two column table the lists the article in one column and a few words or sentences that describe one characteristic of the article.

For each document the list created during coding is sorted by the number of times a key word appears in the document, see Table 1. A weighted point system is used to compare the number of times the word appears across each document. A scale of nine to one points is used with nine points going to the most frequent word, eight to the second etc. If two or more words are found in the document the same number of times it is up to the researcher to decide how they should be ranked. Noah (2013) suggests ranking words with the same number of occurrences by relevance to the issue under investigation. A value for each word is calculated by adding up the points across each document as in Table 2. The top three words are used to define the "essence" of the issue. 
Table 2. Cross Case Analysis

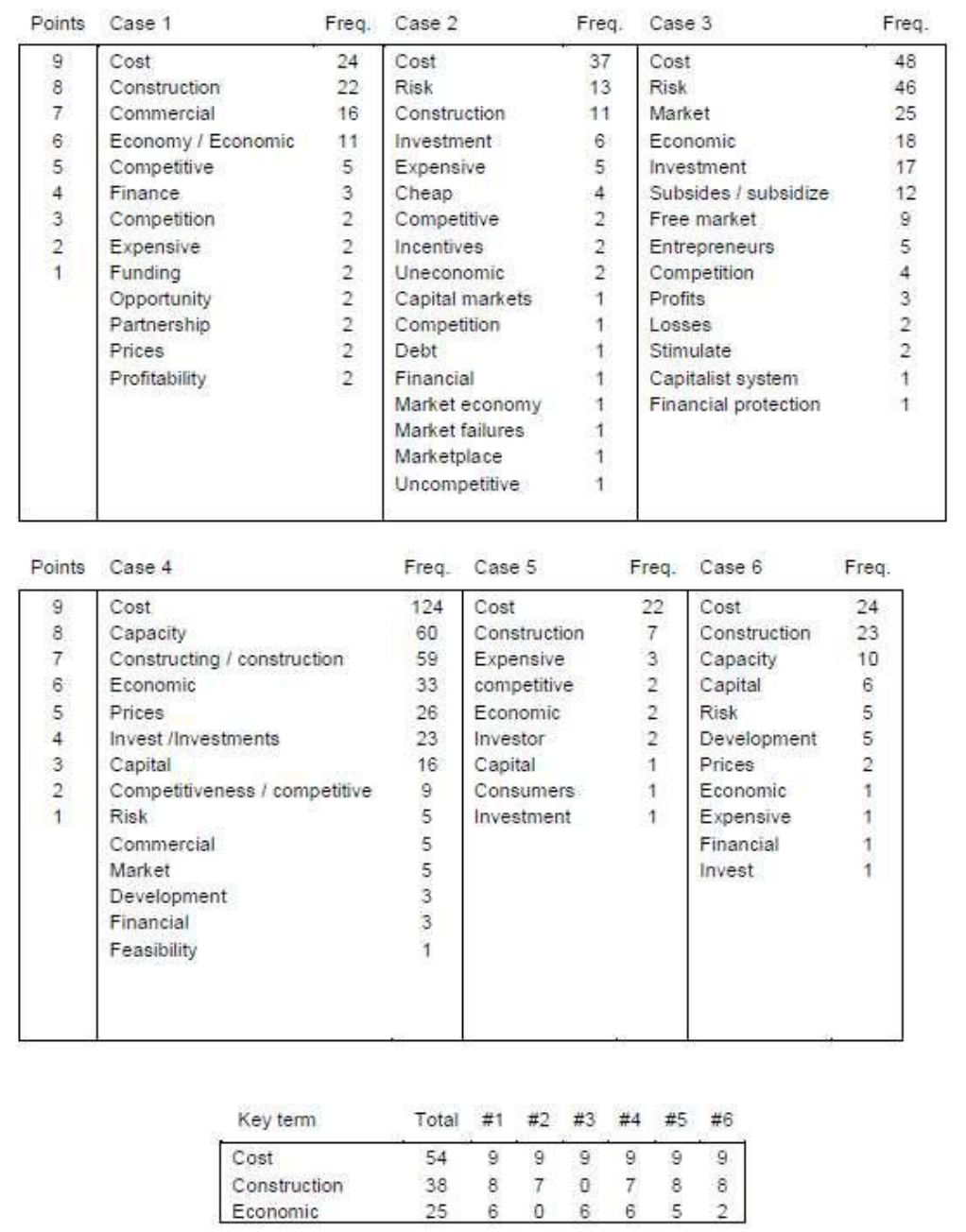

The results of the quaFlitative meta-analysis are the themes and key words from the documents. In Table 1 the themes for nuclear power are Economic, Public Policy, and Technology. The key words for the economic theme would be cost, construction, and economics. The narrative for economics would center around topics such as the cost of construction, government loans and subsidizes, the economic impact to the community and country, cost overruns to name a few.

\section{Presenting results}

The objective of the qualitative meta-analysis is to synthesize and create new knowledge from existing knowledge, it is not meant to be a literature review or to re-hash what has already been written. When presenting the results of the qualitative meta-analysis the researcher should not "boil down" the written word into numeric data, rather he should strive to keep the richness of the original text. Conducting a qualitative meta-analysis requires interpretation and reflectiveness. The numerical "data," in the form of frequency charts and rankings, is meant to be a guide in identifying terms and themes; it is not meant as a means of turning qualitative data into quantitative data. The frequency charts and rankings are best left for the appendix.

When presenting the results the researcher should tell a compelling narrative while keeping the voice of the original documents. The results should express the views of the various stakeholders with quotes from the original documents in order to give all concerned a voice. The narrative should be understandable, structured, and temperate in tone 
(RAND, 2015). The qualitative meta-analysis is meant to inform, explore, and educate, and should not emphasize one point of view over another, it needs to be objective and balanced (RAND, 2015). Objectivity and balance does not mean that the qualitative meta-analysis is just a summary; on the contrary, it should draw conclusions, but only those that are supported by facts and not opinions. The qualitative meta-analysis is an analysis that should be held to the same high academic standards as any other method of research. The conclusions are meant to inform and educate and should be a guide for actionable results, and not just academic theory. The researcher should present the results in a style that is easy to read and is understandable by those outside of academia. Though the qualitative meta-analysis is often published in academic journals the target audience is anyone who has a stake in or interest in the topic being researched.

\section{DISCUSSION AND LIMITATIONS}

\section{Advantages of the qualitative meta-analysis}

The main advantage of the qualitative meta-analysis is the use of secondary data. The data is the set of studies, articles, written accounts, and research documents selected for inclusion. Secondary data of this type is relatively easy to find and retrieve. Most universities, colleges and even some public libraries have access to academic journals and papers in electronic format through subscription services such as EBSCO and ProQuest. The independent researcher can access a sub-set of the same journal articles free with on-line services such as Google Scholar, Acdemia.Net, and Researchgate. The author of this paper completed the research for a doctoral degree using only the on-line resources available through the University library.

By using nonacademic sources such as newspapers, trade journals, dissertations, and unpublished sources, the qualitative meta-analysis reduces the self-selection bias found in some academic fields of study. The outside voices also give the perspectives of underrepresented groups and minority stakeholders thus providing a more holistic view of the subject under investigation. The qualitative meta-analysis is very transparent with the data, in the form of articles and papers, being readily available to other researchers. The reader can decide if the researchers' conclusions are supported by the data.

A qualitative meta-analysis can be completed in a short amount of time and with limited resources. This makes it an appealing methodology for the individual researcher as well as students. It takes less time to find and analyze secondary data than it does for primary data. The data collection phase can be completed in a matter of days instead of weeks or months for primary data. The researcher does not need to develop data collection instruments such as questionnaires or surveys or worry about reaching a statistically significant sample size. Students and non-affiliated independent researchers do not need to deal with the time consuming complexities of having research proposals approved through the Intuitional Review Board process. This however does not relive the researcher from the requirement to conduct their research and present the result in an ethical manner. It is important to give credit and properly cite the sources of secondary data and not to pass it off or imply that it is the researchers own primary data.

\section{Disadvantages of the qualitative meta-analysis}

In the academic world, there is a preference for conducting primary research, and the use of secondary data is seen as being less rigorous or less "scientific." This bias against secondary research can make it harder for researchers using methodologies such as qualitative meta-analysis to get their work published. While this is changing with journals dedicated to qualitative research, quantitative research still dominates. Quantitative research with its measurable results is seen as being more objective than qualitative research. The qualitative meta-analysis relies on the researcher's reflection and interpretation of the data to draw conclusions and results. Even with an audit trial of how and why the interpretations were made the process is still subjective. The bias and personal experiences of the researcher will be a part of the narrative, thus two different researchers can come to different conclusions using the same data.

\section{Further research}

The method of conducting a qualitative meta-synthesis in this paper takes a "top down" approach. It starts with an overarching theme, then smaller "domains" are proposed, followed by finding and comparing key words in each 
article. Further research needs to be conducted in using a "bottom up" approach. In a bottom up approach text analytics could be applied to each article resulting in ranked key words. The key words could then be used to develop domains and overarching themes. The use of text analytics could be supplemented by the use of domain ontologies and automated narrative generation. The result can be validated against the methodology in this paper.

\section{Conclusions}

The qualitative meta-synthesis provides a systematic approach to combining qualitative data in a manner that is reproducible while exploring and better understanding the research topic. New knowledge is created from the synthesis of secondary research data. Themes, ideas, and patterns emerge and are seen only when the individual documents that are part of the synthesis are combined. By taking a systematic approach to the qualitative meta-synthesis the relative importance of the themes and patterns emerge, however the purpose is to examine and understand, not to definitively measure or quantify. The researcher should take care not to try to quantify their results with the use of statistical or Bayesian analysis. The richness and the voices of the documents used should be persevered and present in the presentation of results in a narrative format. When done properly a qualitative meta-synthesis will allow the views of all stakeholders to be heard. The goal of the qualitative meta-synthesis is to create a research study that not only presents the "facts" or "data," but also tells a story that helps to guide policymakers, the public and fellow researchers.

\section{REFERENCES}

Borkovich, D., \& Noah P. (2014). Big Data in the Information Age: Exploring the Intellectual Foundation of Communication Theory. Information Systems Education Journal, 12(1), 15-26.

Corbin-Staton, A. (2009). Contexts of parental involvement: An interpretive synthesis of qualitative literature using the meta-interpretation method. (Order No. 3344879, The George Washington University). ProQuest Dissertations and Theses, , 182.

Creswell, J. (2007). Qualitative inquiry and research design (2nd ed.). Thousand Oaks, CA: Sage Publications.

Finlayson, K., \& Dixon, A. (2008). Qualitative meta-synthesis: A guide for the novice. Nurse Researcher, 15(2), 5971.

Glass, G. V. (1976). Primary, secondary, and meta-analysis of Research1. Educational Researcher, 5(10), 3-8. doi:10.3102/0013189X005010003

Jensen, L. A., \& Allen, M. N. (1996). Meta-synthesis of qualitative findings. Qualitative Health Research, 6(4), 553-553.

Newton, J. D. (2011). How does the general public view posthumous organ donation? A meta-synthesis of the qualitative literature. BMC Public Health, 11(1), 791. doi:10.1186/1471-2458-11-791.

Noah, P. D. (2013). A qualitative meta-analysis of the diffusion of mandated and subsidized technology: United states energy security and independence. (Order No. 3599889, Robert Morris University). ProQuest Dissertations and Theses, 209.

Nonaka, I., \& Konno, N. (1998). The concept of "ba": Building a foundation for knowledge creation. California Management Review, 40(3), 40-54.

RAND (2015). Standards for High-Quality Research and Analysis (CP-413, 5/15). Santa Monica, CA: Corporate publications.

Smith, L. K., Pope, C., Botha, J. L., \& MALTERUD, K. (2005). Patients' help-seeking experiences and delay in cancer presentation: a qualitative synthesis. Commentary. Lancet, 366(9488), 825-831. 
Suri, H. (2011). Purposeful sampling in qualitative research synthesis. Qualitative Research Journal, 11(2), 63-75.

Turner, P. (1997). Secondary analysis of qualitative data. Paper presented at the annual meeting of the American educational research association, Chicago, Il.

Weed, M. (2005). "Meta interpretation": A method for the interpretive synthesis of qualitative research. Forum: Qualitative Social Research, 6(1).

Yin, R. (2009). Case study research design and methods (4th ed.). Thousand Oaks, CA: Sage Publications. 